\title{
USO DE ÁlCOOL E/OU DROGAS: AVALIAÇÃo DOS ASPECTOS DA ESPIRITUALIDADE E RELIGIOSOS
}

\author{
Angelica Martins de Souza Gonçalves ${ }^{1}$ \\ Manoel Antônio dos Santos ${ }^{2}$ \\ Sandra Cristina Pillon ${ }^{3}$
}

O estudo teve por objetivo avaliar aspectos da espiritualidade e religiosos em usuários de álcool e/ou drogas. Trata-se de estudo descritivo exploratório, de abordagem quantitativa. Participaram 138 indivíduos do sexo masculino, vinculados a instituições para reabilitação ou grupo de mútua ajuda, que responderam a um questionário sociodemográfico, AUDIT-C e a versão brasileira da Spirituality Self Rating Scale. Na amostra, 47,1\% eram usuários de álcool e 52,9\% de drogas. Os resultados constataram diferenças entre os grupos em relação aos aspectos religiosos: os usuários de álcool eram católicos $(63,1 \%)$ e não praticantes de religião (50,8\%) e os usuários de drogas eram evangélicos $(43,1 \%)$ e praticavam uma religião (72,2\%). Em relação aos aspectos das práticas espirituais, não foram encontradas diferenças entre os grupos. Ambos demonstraram fazer distinção entre os conceitos de religiosidade e de espiritualidade. Os achados ampliam a compreensão sobre como o enfermeiro pode implementar o cuidado que leva em consideração os aspectos subjetivos do usuário de substâncias psicoativas em sua prática cotidiana.

Descritores: Espiritualidade; Religião e Psicologia; Transtornos Relacionados ao Uso de Substâncias.

\footnotetext{
1 PhD, Professor Adjunto, Departamento de Enfermagem, Universidade Federal de São Carlos, São Carlos, SP, Brasil.

2 PhD, Professor Associado, Faculdade de Filosofia, Ciências e Letras, Universidade de São Paulo, Ribeirão Preto, SP, Brasil.

3 PhD, Professor Titular, Escola de Enfermagem de Ribeirão Preto, Universidade de São Paulo, Centro Colaborador da OMS para o Desenvolvimento da Pesquisa em Enfermagem, Ribeirão Preto, SP, Brasil.
} 


\section{Alcohol ANd/Or DRUG USE: EVAlUATING SPIRITUAL AND RELIGIOUS ASPECTS}

The aim of this study was to evaluate spiritual and religious aspects in drug and/or alcohol users. It is a descriptive, exploratory study using a quantitative approach. A total of 138 men participated, all with links to rehabilitation institutions or support groups, responding to a socio-demographic questionnaire, the AUDIT-C, and to the Brazilian version of the Spirituality Self Rating Scale. In the sample, 47.1\% were alcohol users, 52.9\% drug users. The results demonstrated differences between the groups concerning religious aspects: the alcohol users were Catholics $(63.1 \%)$ or did not practice any religion $(50.8 \%)$ and the drug users were evangelical (43.1\%) or practicing a religion $(72.2 \%)$. With regards aspects of spiritual practice, no differences were found between the groups. Both were shown to distinguish between the concepts of religiosity and spirituality. The findings broadened understanding of how the nurse can implement care which takes into consideration subjective aspects of the psycho-active substance user in their day-to-day practices.

Descriptors: Spirituality; Religion and Psychology; Substance-Related Disorders.

\section{USO DE ALCOHOL Y/O DROGAS: VALIDACIÓN DE LOS ASPECTOS DE ESPIRITUALIDAD Y RELIGIOSOS}

El estudio tiene por objetivo validar aspectos de espiritualidad y religiosos en usuarios de alcohol y/o drogas. Se trata de un estudio descriptivo exploratorio, de abordaje cuantitativo. Participaron 138 individuos de sexo masculino, vinculados a instituciones para rehabilitación o grupo de mutua ayuda, que respondieron a un cuestionario sociodemográfico, AUDIT-C y la versión brasileña de la Spirituality Self Rating Scale. En la muestra, $47,1 \%$ eran usuarios de alcohol y $52,9 \%$ de drogas. Los resultados constataron diferencias entre os grupos en relación a los aspectos religiosos: los usuarios de alcohol eran católicos $(63,1 \%)$ e no practicantes de religión $(50,8 \%)$ y los usuarios de drogas eran evangélicos $(43,1 \%)$ e practicaban una religión $(72,2 \%)$. En relación a los aspectos das prácticas espirituales, no fueran encontradas diferencias entre los grupos. Ambos demostraron hacer distinción entre los conceptos de religiosidad e de espiritualidad. Los resultados amplían la comprensión sobre cómo el enfermero pode implementar o cuidado que leva en consideración los aspectos subjetivos de usuario de substancias psicoactivas en su práctica cotidiana.

Descriptores: Espiritualidad; Religión y Psicología; Trastornos Relacionados con Sustancias.

\section{Introdução}

A importância da religiosidade e da espiritualidade em relação à saúde mental tem sido cada vez mais difundida, constituindo-se em um fenômeno que vem sendo amplamente estudado no âmbito acadêmico, especialmente relacionado às ciências sociais e da saúde ${ }^{(1-3)}$. Nessa perspectiva, estudo de revisão bibliográfica constatou que houve aumento no número de publicações que envolvem os temas espiritualidade e uso de substâncias ${ }^{(4)}$. 
A literatura sugere distinções conceituais entre os termos "espiritualidade" e "religiosidade"(5-7), já que estes têm sido eventualmente interpretados como sinônimos. Uma diferenciação dos termos considera espiritualidade aquilo que dá às pessoas sentido e propósito na vida, enquanto religiosidade é identificada como uma prática sectária ${ }^{(8)}$, parte de um sistema organizado de crenças, práticas, rituais e símbolos criados para facilitar a aproximação do indivíduo às dimensões do sagrado ou transcendente ${ }^{(7)}$.

A valorização crescente dos aspectos da religião e espiritualidade no campo da saúde mental deve-se ao seu reconhecimento como recurso terapêutico disponível para acolher e aliviar o sofrimento humano, que é multidimensional e singular, envolvendo corpo, mente e espírito ${ }^{(1-2,9)}$. Apesar de tal reconhecimento e valorização destes aspectos no campo dos saberes, nas práticas em saúde ainda há pouco interesse na inclusão da dimensão espiritual no cuidado em função do elevado desenvolvimento tecnológico ${ }^{(2)}$.

Com isso, embora seja conhecido o papel da espiritualidade na área da saúde como recurso de bem-estar pessoal, na prática assistencial, ela é negligenciada. Alguns estudos sugerem que os enfermeiros necessitam ter uma perspectiva pessoal/espiritual para poderem oferecer cuidados espirituais $^{(10-12)}$. Esse cuidado pode ser desenvolvido pela aquisição de conhecimentos que auxiliem esses profissionais a adquirir habilidades e competências no cuidado espiritual, de modo a auxiliarem os pacientes a lidar com sua própria espiritualidade ${ }^{(11)}$.

Do ponto de vista do paciente, especialmente daquele em recuperação para dependência química, a relevância disso reside no fato de que elevados níveis de religiosidade e espiritualidade predizem orientações mais otimistas de vida, melhor apoio social, maior resiliência para estresse e menores níveis de ansiedade. A literatura aponta que as práticas espirituais estão associadas à sobriedade, à manutenção da abstinência e à promoção de atitudes que facilitam o processo na entrada da pessoa no tratamento, como recurso de força pessoal ${ }^{(3,13)}$. Por outro lado, os mecanismos de influência da espiritualidade sobre tais processos de recuperação ainda são pouco conhecidos devido à sua complexidade ${ }^{(4,14)}$.
Ademais, estudos de revisão sobre espiritualidade na área da dependência química concluíram que esse constructo é frequentemente definido e mensurado de forma precária ${ }^{(4,14)}$. Adeptos de programas direcionados à espiritualidade, entretanto, concordam que a crença em um poder superior é fundamental para o processo de mudança do comportamento, especialmente o uso de álcool e/ou outras drogas ${ }^{(14-15)}$.

A espiritualidade, como dimensão pessoal, permeia toda a história de vida do indivíduo, enquanto a religiosidade se refere a um meio que possibilita a manifestação dos aspectos espirituais (relacionados ou não ao tratamento). Nesse contexto, apesar de muitas pessoas recorrerem a recursos espirituais para recuperação da dependência, a vinculação a uma religião geralmente ocorre em um momento posterior, em decorrência dos sucessos observados em terceiros ou em sua própria recuperação paulatina ${ }^{(3)}$.

Diversos cientistas do início do século $\mathrm{XX}$ estavam convencidos de que, com o desenvolvimento da ciência e da racionalidade moderna, a religião estaria fadada ao desaparecimento. Porém, o que ocorreu foi a valorização de aspectos subjetivos do indivíduo, que busca respostas às questões fundamentais da vida por meio de um relacionamento com o transcendente, um fenômeno denominado "transmutação de religião em espiritualidade"(16).

Considerando a necessidade de melhor compreensão da temática nos diversos contextos de atuação da enfermagem, o presente estudo teve por objetivo identificar o uso de álcool e/ou de outras drogas e avaliar os aspectos da espiritualidade e religiosos em usuários de instituições de reabilitação e grupo de mútua ajuda.

\section{Método}

Trata-se de um estudo descritivo exploratório, de abordagem quantitativa.

A amostra foi composta por 138 usuários de substâncias psicoativas vinculados a instituições de reabilitação e grupo de mútua ajuda de quatro municípios do interior paulista, sendo: uma Comunidade Terapêutica CT (de cunho evangélico, católico, sem vinculação religiosa), um Centro de Atenção Psicossocial Álcool e Drogas (CAPSad) e um grupo de Alcoólicos Anônimos (AA). 
Este estudo é derivado de uma dissertação de mestrado ${ }^{(17)}$, que também deu origem ao estudo de validação da versão brasileira da Spirituality Self Rating Scale (SSRS-br) ${ }^{(18)}$. Foram critérios de inclusão na amostra: ter idade igual ou superior a 18 anos; estar vinculado a Comunidades Terapêuticas (CTs) ou grupo de mútua ajuda (Alcoólicos Anônimos - AA). Foram critérios de exclusão: ser analfabeto ou não apresentar condição de compreensão dos instrumentos de pesquisa.

Os dados foram coletados no período de agosto a outubro de 2007. Foi utilizado um instrumento autoaplicado, dividido em duas partes, contendo informações sociodemográficas, questões relacionadas ao uso de drogas, Teste de Identificação do Uso de Álcool versão C (AUDIT-C) e a SSRS-br ${ }^{(18)}$.

O AUDIT-C é um questionário composto por três perguntas (número de doses, frequência de uso e consumo de cinco ou mais doses em uma única ocasião). Para leitura dos níveis de risco do consumo é necessário fazer o somatório das respostas, que varia de zero a 12 pontos, sendo diferenciada a pontuação para os sexos. Para o sexo masculino, escore a partir de cinco pontos indica um consumo nocivo e direciona a intervenção ${ }^{(19)}$.

A SSRS é uma escala composta por seis itens (unidirecional) que visam a mensurar o quanto o sujeito considera ou julga importantes questões pertinentes à sua dimensão espiritual e as aplica em sua vida. Validada em usuários de substâncias psicoativas, apresenta propriedades psicométricas satisfatórias, com coeficiente de alfa de Cronbach global de 0,83, variando de 0,70 a $0,88^{(18)}$. Os itens que compõem a SSRS consideram práticas religiosas e preceitos teóricos de Programas baseados em 12 passos. As respostas são do tipo Likert Scale e variam de "1=concordo totalmente" a " $5=$ discordo totalmente". Para cálculo dos escores, deve-se fazer uma recodificação de cada item (ex.: $5=1 ; 4=2 ; 3=3 ; 2=4$; $1=5)$, obtendo-se o valor total. Quanto maior é o valor identificado, maior o nível de orientação espiritual.

Os dados foram inseridos no Statistical Package for Social Sciences (SPSS), versão 10. Para avaliar os aspectos religiosos entre os grupos de usuários de álcool e/ou de drogas foram aplicados o teste qui-quadrado e o teste $t$ de Student para avaliar as diferenças entre as médias dos escores da idade, idade do primeiro uso, da SRSS-br e do AUDIT-C entre os grupos avaliados. Foi utilizado ainda o teste de KruskalWallis para avaliar o ranking das médias da pontuação da SRSS e do AUDIT-C com as variáveis sociodemográficas e os aspectos religiosos. O nível de significância adotado foi de 5\%.

Este estudo foi aprovado pelo Comitê de Ética em Pesquisa da Escola de Enfermagem de Ribeirão Preto da Universidade de São Paulo, Processo $\mathrm{n}^{-}$0804. Todos os participantes foram orientados sobre a pesquisa e assinaram o Termo de Consentimento Livre e Esclarecido.

\section{Resultados}

Informações sociodemográficas

A amostra do estudo foi composta por 138 adultos do sexo masculino, com média de idade de 39 anos (desvio-padrão $\pm 13,60$ ), variando entre 18 e 69 anos. Desses participantes, 81 $(58,7 \%)$ eram vinculados às CTs, $30(21,7 \%)$ ao AA e 27 (19,6\%) ao CAPS-ad; 87 (63\%) viviam sozinhos, $64(46,4 \%)$ tinham ensino médio (completo ou incompleto), 95 (68,8\%) tinham emprego formal, $65(47,1 \%)$ trabalharam em tempo integral no último ano. Todos os usuários que frequentavam os locais da pesquisa no período de coleta foram incluídos no estudo.

Em relação ao tipo de droga usada, identificou-se que 65 (47,1\%) eram usuários de álcool e $73(52,9 \%)$ de drogas. No grupo de usuários de álcool não foi identificado o uso de outras drogas, no entanto, no outro grupo constatou-se um número expressivo de usuários de cocaína, maconha e álcool. Também foi observada diferença entre os grupos avaliados, com destaque para o grupo de usuários de drogas, nas variáveis: tipo de droga que o levou a buscar tratamento, tempo em tratamento, local e histórico de internação. Essas diferenças foram estatisticamente significantes. Os resultados podem ser apreciados na Tabela 1.

Quanto aos aspectos religiosos, foram constatadas diferenças entre os grupos: em relação aos usuários de álcool, $41(63,1 \%)$ eram afiliados à religião católica, $33(50,8 \%)$ não praticantes da 
religião e $23(35,4 \%)$ frequentavam apenas aos finais de semana. Quanto aos usuários de drogas, $31(43,1 \%)$ pertenciam à religião evangélica, 52 $(72,2 \%)$ praticavam a religião, $34(47,2 \%)$ frequentavam sessões religiosas todos ou quase todos os dias. Ambos os grupos pertenciam a uma família praticante e não consideravam religiosidade sinônimo de espiritualidade (Tabela 2).

A Tabela 3 evidencia que houve diferença estatisticamente significante entre os grupos de usuários de álcool e de drogas no que concerne à idade de início do uso de drogas e a pontuação do AUDIT-C. Embora os usuários de álcool apresentem médias de idade e pontuação na SRSSbr maiores quando comparadas às dos usuários de drogas, as diferenças obtidas não alcançaram significância estatística.

As médias dos escores da SRSS-br foram maiores no grupo de usuários de drogas atendidos no CAPS-ad e entre os que trabalhavam em tempo parcial. No entanto, as diferenças não foram estatisticamente significantes.
As demais variáveis, quando comparadas as médias dos escores da SRSS-br e do AUDIT-C com as variáveis sexo, idade, estado civil, religião e tipo de drogas usadas, não apresentaram diferenças significantes. Também não houve diferença estatisticamente significante entre idade, escores da SRSS e do AUDIT-C.

Não foram encontradas diferenças entre as médias dos escores da SRSS-br dos usuários que tinham familiar praticante de religião e aqueles que afirmaram ter familiar não praticante, embora a média dos escores da SSRS tenha sido maior no grupo de usuários com familiar praticante.

Quanto ao nível de gravidade do consumo do álcool, aferida pelo AUDIT-C, as maiores pontuações foram obtidas entre os que não trabalhavam e os usuários do CAPS-ad, com diferenças estatísticas significativas.

Os usuários que consideraram espiritualidade sinônimo de religiosidade apresentaram pontuação maior na SSRS-br, mas a diferença entre os grupos não foi estatisticamente significante.

Tabela 1 - Distribuição numérica e percentual dos usuários de álcool e de outras drogas em função dos aspectos relacionados ao uso e tratamento. $(\mathrm{N}=138)$

\begin{tabular}{|c|c|c|c|c|c|}
\hline & \multicolumn{2}{|c|}{ Usuários de álcool } & \multicolumn{2}{|c|}{ Usuários de drogas } & \multirow{2}{*}{ Valores de $p$} \\
\hline & $\mathbf{N}$ & $\%$ & $\mathbf{N}$ & $\%$ & \\
\hline \multicolumn{6}{|l|}{ Tipo de droga usada } \\
\hline \multicolumn{6}{|l|}{ Álcool } \\
\hline Não & - & - & 41 & 56,2 & 000 \\
\hline Sim & 65 & 100 & 32 & 43,8 & \\
\hline \multicolumn{6}{|l|}{ Maconha } \\
\hline Não & 65 & 100 & 34 & 46,6 & \multirow{2}{*}{,000 } \\
\hline Sim & - & - & 39 & 53,4 & \\
\hline \multicolumn{6}{|l|}{ Cocaína } \\
\hline Não & 65 & 100 & 11 & 15,1 & \multirow{2}{*}{, 000} \\
\hline Sim & - & - & 62 & 84,9 & \\
\hline \multicolumn{6}{|c|}{ Tipo de droga que o levou a buscar ajuda } \\
\hline Álcool & 65 & 100 & - & - & \multirow{4}{*}{000} \\
\hline Maconha & - & - & 4 & 5,5 & \\
\hline Cocaína ou crack & - & - & 25 & 34,2 & \\
\hline Múltiplas drogas & - & - & 44 & 60,3 & \\
\hline \multicolumn{6}{|l|}{ Tempo de tratamento } \\
\hline$\leq 30$ dias & 30 & 50,8 & 51 & 72,9 & \multirow{3}{*}{,009 } \\
\hline Entre 31 e 60 dias & 7 & 11,9 & 9 & 12,9 & \\
\hline$>60$ dias & 22 & 37,3 & 10 & 14,3 & \\
\hline \multicolumn{6}{|l|}{ Local } \\
\hline CTs & 26 & 40,0 & 55 & 75,3 & \multirow{3}{*}{,000 } \\
\hline$A A$ & 23 & 35,4 & 7 & 9,6 & \\
\hline CAPS-ad & 16 & 24,6 & 11 & 15,1 & \\
\hline \multicolumn{6}{|l|}{ Histórico de internação } \\
\hline Sim & 47 & 73,4 & 71 & 97,3 & \multirow{2}{*}{, 000} \\
\hline Não & 17 & 26,6 & 2 & 2,7 & \\
\hline
\end{tabular}

$\mathrm{p}<0,05$, teste de Qui-quadrado 
Tabela 2 - Distribuição numérica e percentual dos usuários de álcool e de outras drogas. $(\mathrm{N}=138)$

\begin{tabular}{|c|c|c|c|c|c|}
\hline & \multicolumn{2}{|c|}{ Usuários de álcool } & \multicolumn{2}{|c|}{ Usuários de drogas } & \multirow{2}{*}{ Valores de $p$} \\
\hline & $\mathbf{N}$ & $\%$ & $\mathbf{N}$ & $\%$ & \\
\hline \multicolumn{6}{|l|}{ Afiliação religiosa } \\
\hline Católica & 41 & 63,1 & 30 & 41,7 & \multirow{4}{*}{, 000} \\
\hline Evangélica & 7 & 10,8 & 31 & 43,1 & \\
\hline Espírita & 5 & 7,7 & 5 & 6,9 & \\
\hline Não tem & 12 & 18,5 & 6 & 8,3 & \\
\hline \multicolumn{6}{|l|}{ Prática de religião } \\
\hline Sim & 32 & 49,2 & 52 & 72,2 & \multirow{2}{*}{, 005} \\
\hline Não & 33 & 50,8 & 20 & 27,8 & \\
\hline \multicolumn{6}{|l|}{ Frequência da prática religiosa } \\
\hline Todos ou quase todos os dias & 12 & 18,5 & 34 & 47,2 & \multirow{4}{*}{,001 } \\
\hline Somente aos finais de semana & 23 & 35,4 & 23 & 31,9 & \\
\hline Raramente & 21 & 32,3 & 8 & 11,1 & \\
\hline Nenhuma & 9 & 13,8 & 7 & 9,7 & \\
\hline \multicolumn{6}{|l|}{ Família praticante de religião } \\
\hline Sim & 54 & 83,1 & 61 & 83,6 & \multirow{2}{*}{, 559} \\
\hline Não & 11 & 16,9 & 12 & 16,4 & \\
\hline \multicolumn{6}{|c|}{ Considera espiritualidade com significado de religiosidade ou religião } \\
\hline Sim & 30 & 46,2 & 23 & 31,5 & \multirow{2}{*}{,056 } \\
\hline Não & 35 & 53,8 & 50 & 68,5 & \\
\hline
\end{tabular}

$\mathrm{p}<0,05$, teste de Qui-quadrado

Tabela 3 - Diferença entre as médias da idade atual, idade de início do uso, pontuação do AUDIT-C e SRSS-br. $(\mathrm{N}=138)$

\begin{tabular}{|c|c|c|c|c|}
\hline Grupo de usuários & Média & $\mathrm{Dp}$ & Erro-padrão das médias & Valores de $p$ \\
\hline \multicolumn{5}{|l|}{ Idade atual } \\
\hline Álcool & 48,1 & 9,7 & 1,2 & \multirow[t]{2}{*}{,177 } \\
\hline Drogas & 30,8 & 11,2 & 1,4 & \\
\hline \multicolumn{5}{|c|}{ Idade de início do uso de drogas } \\
\hline Álcool & 18,5 & 8,6 & 1,3 & \multirow{2}{*}{,000 } \\
\hline Drogas & 15,3 & 4,4 &, 5 & \\
\hline \multicolumn{5}{|l|}{ AUDIT-C } \\
\hline Álcool & 7,3 & 5,0 & 6 & \multirow{2}{*}{,039 } \\
\hline Drogas & 7,9 & 4,4 &, 5 & \\
\hline \multicolumn{5}{|l|}{ SRSS-br } \\
\hline Álcool & 12,6 & 5,4 & ,6 & \multirow{2}{*}{ 159, } \\
\hline Drogas & 11,6 & 4,6 &, 5 & \\
\hline
\end{tabular}

$\mathrm{p}<0,05$, Teste $\mathrm{t}$ de Student.

\section{Discussão}

Com este estudo buscou-se uma melhor compreensão da temática sobre o uso de álcool e/ou de outras drogas e os aspectos de espiritualidade/religiosos em usuários de instituições de reabilitação e grupo de mútua ajuda.

As características sociodemográficas da amostra foram convergentes com outros estudos da literatura, predominando usuários do sexo masculino, adultos, com baixo nível de escolaridade $^{(3,7-8,14,19)}$.
Quanto ao uso de substâncias psicoativas, foram constatadas diferenças entre os grupos avaliados. Os usuários de álcool não utilizavam outros tipos de drogas e estavam vinculados em sua maioria às CTs $(40 \%)$, enquanto os usuários de drogas eram poliusuários $(60 \%)$ também vinculados às CTs $(75,3 \%)$, e que já haviam passado por internação $(97,3 \%)$.

Esses dados sugerem que usuários de drogas estão recorrendo a serviços de internação integral como um recurso de tratamento que os afasta do convívio social e, consequentemente, 
das condições ambientais que favorecem a manutenção do comportamento de uso das substâncias. Com efeito, nas últimas décadas tem havido uma expansão da utilização das CTs. Trata-se de um modelo de tratamento com características diferentes dos modelos tradicionais, que utiliza o ambiente e o próprio usuário na promoção do aprendizado e das mudanças necessárias para romper com o ciclo da dependência, que envolve o uso, abstinência e recaída ${ }^{(8,20)}$.

$\mathrm{Na}$ avaliação dos aspectos religiosos (afiliação, prática, frequência), entre os usuários observou-se que os que faziam uso de álcool eram afiliados à religião católica, não praticavam a religião e frequentavam com menor intensidade a religião, quando comparados aos usuários de drogas. A religião proporciona a muitos usuários apoio social, assim como um esquema cognitivo clinicamente efetivo que contribui para reduzir o estresse e incrementar o bem-estar subjetivo ${ }^{(2,4)}$ em dependentes de álcool e/ou de outras drogas em processo de reabilitação ${ }^{(3,6)}$.

Estudos apontam que o estímulo ao exercício da dimensão espiritual tem potencial para facilitar o processo de reabilitação para abuso e dependência de álcool e/ou de outras drogas, apesar de seus mecanismos de ação serem ainda pouco conhecidos. Isso pode estar relacionado à adoção de um novo estilo de vida, no qual vínculos, práticas cotidianas e até mesmo a adesão a determinada religião são incentivados para que os indivíduos consigam esquivar-se de situações que favoreçam o uso de substâncias psicoativas ${ }^{(15,17)}$.

Os achados deste estudo mostraram que espiritualidade independe dos aspectos da vida religiosa dos indivíduos, seja em relação à prática e frequência a cerimoniais religiosos, no âmbito pessoal ou familiar, seja em relação à doutrina religiosa de escolha, corroborando os achados da literatura $^{(4,6-7)}$.

Esses resultados trazem implicações para a prática de qualidade do cuidado integral. $\mathrm{O}$ enfermeiro deve estar atento à dimensão religiosa e espiritual, respeitando os valores e crenças dos usuários de serviços, entendendo que a espiritualidade é parte integral das necessidades humanas e uma dimensão essencial para o processo de recuperação ${ }^{(1,9-10)}$.

$\mathrm{O}$ fato de a religiosidade não ser entendida como sinônimo de espiritualidade não se mostrou significativo na amostra investigada, diferentemente do que aponta a literatura ${ }^{(6-7)}, 0$ que é auspicioso porque sugere que o exercício da dimensão espiritual pode ser trabalhado em qualquer âmbito de tratamento.

A espiritualidade identificada entre os usuários de substâncias psicoativas, de acordo com as médias identificadas neste estudo, parece estar relacionada à busca de uma vida mais estável e equilibrada, talvez pelo reconhecimento de que o sentimento pessoal possa estimular um desfecho positivo quanto ao enfrentamento do problema, facilitando a manutenção do estado de abstinência, melhorando os relacionamentos interpessoais e ajudando a tolerar sentimentos disruptivos, como a ansiedade. Estudo voltado para outros problemas de saúde, que não envolvem a dependência de drogas, aponta que um número relevante de pacientes considera suas necessidades espirituais importantes e gostariam de receber alguma atenção nessa dimensão ${ }^{(15)}$. No âmbito da dependência química, a espiritualidade pode estar relacionada à redução do uso e, para os casos em tratamento, tem sido uma ferramenta terapêutica importante na redução de episódios de craving, melhoria no nível de saúde e incremento da qualidade de vida ${ }^{(7)}$.

Dentre as limitações do presente estudo podem-se mencionar as dificuldades em mensurar a religiosidade, devido à polissemia desse conceito, sobretudo quando é avaliado por meio de variáveis como afiliação (por exemplo, católica, evangélica), prática religiosa (praticante/não praticante) ou frequência de comparecimento a cultos religiosos (semanal, mensal). Essas estratégias de avaliação são consideradas limitadas por restringirem o estudo de algo tão complexo como a religiosidade na vida de um paciente a uma única variável.

Outra dificuldade encontrada refere-se à diferenciação dos conceitos de religiosidade e espiritualidade, uma vez que as pessoas tendem a atribuir diferentes significados a esses constructos. Isso impõe um desafio ao profissional enfermeiro que, em sua prática assistencial, deve estar atento para essa multiplicidade semântica. Historicamente, aspectos da religiosidade e da espiritualidade estão presentes no discurso, no ensino e nos pressupostos do exercício da assistência de enfermagem ${ }^{(1,12)}$. Apesar disso, os 
profissionais da área nem sempre possuem habilidades para atender tais dimensões, em parte pela necessidade de melhor compreensão da complexidade dos conceitos e por questionamentos sobre o modo de se programar esse cuidado nas práticas clínicas ${ }^{(1-10)}$.

Em relação ao instrumento utilizado para mensurar espiritualidade, existem diferenças subjetivas de entendimento do que é e de como se pode exercê-la. Finalmente, o tipo de amostra utilizada é representativa apenas para os locais investigados, não sendo possível extrapolar e generalizar os resultados para outros contextos.

\section{Conclusão}

Este estudo mostrou que há uma relação complexa entre aspectos religiosos e espirituais em usuários de substâncias psicoativas. Houve diferença estatisticamente significante nos aspectos religiosos entre o grupo de usuários de álcool e o grupo de drogas; no entanto, a espiritualidade e os conceitos de religiosidade e espiritualidade utilizados não se diferenciaram entre os grupos. Esses resultados têm implicações importantes no âmbito do tratamento e da reabilitação psicossocial, pois religião e espiritualidade podem funcionar como elementos de desfecho positivo dentre os fatores de proteção ao uso de drogas. Desse modo, os achados obtidos oferecem subsídios para que o cuidado holístico possa se tornar efetivo na prática cotidiana do enfermeiro, ampliando a compreensão sobre como este profissional pode implementar o cuidado que leva em consideração os aspectos subjetivos do usuário de substâncias psicoativas.

\section{Referências}

1. Draper P. An integrative review of spiritual assessment: implications for nursing management. J Nurs Manag. 2012 Dec;20(8):970-80.

2. Mohr K. Spiritual issue in psychiatric care. Perspect Psychiatr C. 2006 Aug;42(3):174-83.

3. Sanchez ZM, Nappo SA. A religiosidade, a espiritualidade e o consumo de drogas. Rev Psiquiatr Clín. 2007;34(supl 1):73-81.

4. Cook CCH. Addiction and spirituality. Addiction. 2004;99(3):539-51.
5. Guimarães HP, Avezum A. O impacto da espiritualidade na saúde física Rev Psiquiatr Clín. 2007;34(supl 1):88-94.

6. Clarke JA. Discussion paper about "meaning" in the nursing literature on spirituality: an interpretation of meaning as "ultimate concern" using the work of Paul Tillich. Int J Nurs Stud. 2006;43(7):915-21.

7. Moreira-Almeida A, Koening HG. Retaining the meaning of the words religiousness and spirituality: a commentary on the WHOQOL SRPB group's "a cross-cultural study of spirituality, religion, and personal beliefs as components of quality of life". Soc Sci Med. 2006;63(4):843-5. 8. Galanter M, Dermatis H, Bunt G, Willians C, Trujillo M, Steinke P. Assessment of spirituality and its relevance to addiction treatment. J Subst Abuse Treat. 2007;3(33):257-64.

9. Monod S, Brennan M, Rochat E, Martin E, Rochat S, Bula CJ. Instruments measuring spirituality in clinical research: a systematic review. J Gen Intern Med. 2011;26(11):1345-57.

10. Chung LYF, Wong FK, Chan MF. Relationship of nurses' spirituality to their understanding and practice of spiritual care. J Adv Nurs. 2006 April;58(2):158-70.

11. Narayanasamy A. Nurses'awareness and educational preparation in meeting their patients' spiritual needs. Nurse Educ Today. 1993 Jun;13(3):196-201.

12. Greenstreet WM. Teaching spirituality in nursing: a literature review. Nurse Educ Today. 1999;19(8):649-58.

13. Poage ED, Ketzenberger KE, Olson J. Spirituality, contentment, and stress in recovering alcoholics. Addict Behav. 2004;29(9):1857-62.

14. Lillis J, Gifford E, Humphreys K, Moos R. Assessing Spirituality/Religiosity in the Treatment Environment: The Treatment Spirituality/Religiosity Scale. J Subst Abuse Treat. 2008;35(4):427-33.

15. Arnold RM, Avants K, Margolin A, Marcotte

D. Patient attitudes concerning the inclusion of spirituality into addiction treatment. J Subst Abuse. 2002;23:319-26.

16. Siqueira D. O labirinto religioso ocidental: da religião à espiritualidade, do institucional ao não convencional. Soc e Estado. 2008;23(2):425-62. 17. Gonçalves AMS. Estudo dos níveis motivacionais em relação ao uso de substâncias 
psicoativas e a espiritualidade. [dissertação]. Ribeirão Preto: Escola de Enfermagem de Ribeirão Preto da Universidade de São Paulo; 2008.

18. Gonçalves AMS, Pillon SC. Adaptação transcultural e avaliação da consistência interna da versão em português da Spirituality Self Rating Scale (SSRS). Rev Psiquiatr Clín. 2009;36(1):10-5.

19. Meneses-Gaya C, Zuardi AW, Loureiro SR, Hallak JE, Trzesniak C, de Azevedo Marques $\mathrm{JM}$, et al. Is the full version of the AUDIT really necessary? Study of the validity and internal construct of its abbreviated versions. Alcohol Clin Exp Res. 2010 Aug;34(8):1417-24.

20. Fracasso L. Comunidades Terapêuticas. In: Diehl A, Cordeiro DC, Laranjeira R, organizadores. Dependência química. Porto Alegre: Artmed; 2011. p. 61-9. 